\title{
Influence of type and duration of training on the presence of an abnormal ECG in high- performance athletes
}

\author{
Geoffrey Verrall, ${ }^{\oplus 1}$ Angus Hains, ${ }^{2}$ Bronte Ayres, ${ }^{2}$ Richard Hillock ${ }^{2}$
}

'Department of Sports Medicine, South Australian Sports Institute, Brooklyn Park, South Australia, Australia

${ }^{2}$ Department of Sports Medicine, SA Heart, Adelaide, South Australia, Australia

\section{Correspondence to} Dr Geoffrey Verrall, South Australian Sports Institute, Brooklyn Park, SA 5032, Australia; geoffrey.verrall@ gmail.com

Received 12 September 2018 Revised 31 January 2019 Accepted 9 February 2019

\section{Check for updates}

(c) Author(s) (or their employer(s)) 2019. No commercial re-use. See rights and permissions. Published by BMJ.

To cite: Verrall G, Hains A, Ayres $\mathrm{B}$, et al. Heart Asia 2019;11:e011120. doi:10.1136/

heartasia-2018-011120

\section{ABSTRACT}

Objective To undertake an ECG in high level athletes to determine the morphology of the ECG and to assess the influences on the ECG of the amount of time in a highperformance programme and the type of sport played.

Design Cross-sectional cohort study.

Setting High-performance sports programme. Interventions Current symptoms (questionnaire) and length of time in a high-performance sports programme were recorded. Sports were classified as either high maximal oxygen uptake continuous, high maximal oxygen uptake repeated effort or static. An ECG was performed and classified by 2010 European Society of Cardiology guidelines into Groups 0 (normal), Group 1 (common and training-related ECG changes) and Group 2 (uncommon and training unrelated ECG changes).

Results The following were recorded: length of time in high performance sport programme (mean 2.3 years), type of sport (Continuous 103, Repeated effort 133, Static 37), ECG changes Group 0 ( $n=83,31 \%)$, Group $1(n=173,63 \%)$ and Group $2(n=17,6.2 \%)$. Athletes with an increased length of time in a high performance programme demonstrated a higher likelihood of Group 2 ECG changes when compared with Groups 0 and 1 $(p=0.05)$. The questionnaire did not help detect athletes with Group 2 ECG changes.

Conclusions This study demonstrates that an increased length of time in a high performance programme was associated with an increased number of detectable Group 2 ECG changes. Overall, the further investigation rate was $6.2 \%$.

\section{INTRODUCTION}

Sudden cardiac death (SCD) is the leading cause of mortality for young athletes on the playing field. ${ }^{1}$ Since 1996, the American Heart Association has recommended preparticipation evaluation of an athlete to try and identify those that may have structural and/or electrical heart abnormalities that increase the risk for SCD during activity. ${ }^{2}$ In addition to a medical history and a physical examination, a 12-lead ECG is recommended as part of this preparticipation evaluation. ${ }^{34}$ Athletic training will cause the myocardium to undergo physiological adaptation to exercise and these adaptations may cause changes to the resting ECG. ${ }^{5}$ The challenge in interpreting ECGs in the athletic population is to determine if changes are due to physiological adaptation or underlying cardiac pathology that may lead to an increased risk of SCD such as cardiomyopathy. ${ }^{6}$

Athletes with abnormal ECGs undergo further cardiac investigation but few are found to have a
Key messages

What is already known about this subject?

- ECG criteria for active athletes have been developed so that unnecessary investigation can be minimised. These criteria are continuously being tested for robustness.

What does this study add?

- The European Society of Cardiology classification of uncommon and training unrelated ECG changes is not as robust when length of time in a high-performance programme and increased intensity of sport as measured by maximal oxygen uptake are taken into account.

How might this impact on clinical practice?

- Continuing development of ECG criteria for active athletes needs to occur.

significant and serious underlying cardiac abnormality that would preclude them from participation in sport. ${ }^{6}$ The original ECG studies had a further investigation rate due to a detectable perceived ECG abnormality in $15 \%$ of athletes. ${ }^{1}$ More recent studies have demonstrated a significant reduction in this follow-up rate as clinicians have considered that more of these detected ECG changes are in actual fact normal adaptive changes seen in the athletic population. ${ }^{7-9}$

While it is important that those at risk are identified, it is equally important that the tools used for screening must be cost effective and specific enough to avoid preventing otherwise normal athletes from exercise and competition. In response to these considerations, the European Society of Cardiology (ESC) consensus statement was released in 2010 and categorised athlete ECG findings into Groups: 0 (normal ECG), 1 (common and training-related ECG changes), 2 (uncommon and training unrelated ECG changes), respectively. ${ }^{10}$ Athletes with Group 2 changes were recommended to have further investigation to look for any possible structural and/ or electrical abnormality that may predispose the athlete to SCD. ${ }^{10}$ At the time of undertaking this study, the performance of the 2010 ESC criteria had not yet been fully assessed but one retrospective study of 508 American university athletes demonstrated a reduction of ECG abnormalities from 16\% using the 2005 ESC criteria to $9.6 \%$ using the ESC 2010 criteria. $^{11}$

The aim of this study was to assess and categorise the ECG findings in a group of young Australian 
high-performance athletes with respect to the ESC 2010 guidelines for Groups 0, 1 and 2 to determine the relevant follow-up rate for further investigation. A further aim of this study was to see if the ESC group categories would remain robust when the number of years in a high performance programme and type of sport categorised on the basis of the physiological demand of the sport are taken into account especially in relation to uncommon and training unrelated ECG changes.

\section{METHOD}

Approval for this study was given by the Australian Institute of Sport (AIS) ethics committee. Participation in the study was voluntary and all athletes gave signed informed consent before involvement in the study. All subjects were athletes in high-performance sports programmes as scholarship holders at the South Australian Sports Institute (SASI). Thus, our study was cross-sectional in design with athletes having been in sports programmes for differing amounts of time being studied. Sports were classified by their physiological profile taking into account the energy system the sports most targeted in their training and playing regimes. Three sport type classifications, based on the Mitchell classification $^{12}$ were made (1) performance occurring at a high percentage of maximal oxygen uptake (VO2max) in a continuous nature (continuous), (2) performance occurring at a high VO2max with repeated sprint and/or jumping efforts (repeated effort) and (3) performance at a lower percentage of VO2max (static). Athlete information obtained included: age, sex, ethnicity, sport and length of time (measured in years) in a high performance sport programme. Entrance athletes were classified as being in a high-performance programme for less than 1 year.

All athletes then answered a 9 question cardiac questionnaire (online supplementary appendix 1). The answers were categorised into: 0 (all questions answered no), 1 (one question answered yes) or greater than 1 (more than one question answered yes).

The answers were further classified into possible cardiac symptoms or possible predisposition to abnormality by history. These symptoms isolated by the questionnaire included dizziness and chest pain with exercise, abnormal heart rate, palpitations, chest tightness and coughing spells (those athletes who had asthma were excluded to avoid confounding from these responses).

A 12 lead ECG was performed by a qualified heart technician. This ECG was then analysed by a sports physician and three cardiologists (all blinded to the history) using the ESC 2010 guidelines. Athletes were then classified into three groups according to their ECG: Group 0 (no abnormality), Group 1 (common and training-related ECG changes) and Group 2 (uncommon and training unrelated ECG changes). All athletes with Group 2 changes had subsequent appropriate cardiac investigations including echocardiogram.

Athletes were then categorised and analysed based on: (1) number of positive answers to the questions in the questionnaire $(0,1$ or greater than 1$),(2)$ type of sport (Continuous, Repeated effort or Static) and (3) ECG changes (Groups 0, 1 and 2).

A Kruskal-Wallis test was performed for comparing the three ECG groups (Groups 0, 1 and 2) with adjustment for age and based on ranked one-way analysis of covariance. For comparing two groups (Groups 0 and 1), Mann-Whitney $U$ test were performed. A Pearson $X$ test was used when comparing the three ECG group changes with the three classified types of sports. To adjust for age or length of time in programme for all of these parameters, we used multinomial logistic regression.
Table 1 Classification of sports on the basis of physiological demands and predominant energy system used based on playing and training regimes

\begin{tabular}{|c|c|}
\hline Type of sport & Sport (numbers in brackets) \\
\hline Continuous (103) & $\begin{array}{l}\text { Long distance athletics (2), Cycling (25), Kayaking (29), } \\
\text { Rowing (19), Swimming (15), Water Polo* (13) }\end{array}$ \\
\hline Repeated effort (133) & $\begin{array}{l}\text { Sprint athletics (4), Basketball (21), Beach Volleyball* (11), } \\
\text { BMX cycling (2), Football (soccer) (11), Field Hockey (22), } \\
\text { Netball (38), Volleyball }{ }^{*}(24)\end{array}$ \\
\hline Static (37) & $\begin{array}{l}\text { Baseball (10), Bowling (1), Diving (6), Equestrian (4), } \\
\text { Gymnastics (2), Judo (1), Shooting (6), Trampoline (6), } \\
\text { Weightlifting (1) }\end{array}$ \\
\hline
\end{tabular}

(1) Continuous=performance occurs at a high percentage of maximal oxygen uptake with predominately a continuous consistent effort. (2) Repeated effort=performance occurs at a high percentage of maximal oxygen uptake with repeated sprinting and/or jumping efforts and (3) Static=performance occurs at a lower percentage of maximal oxygen uptake and generally of a static nature.

*Principally the sports were classified on the basis of the training regime implemented. Thus, the author has confidence in the classification of water polo in predominately continuous and volleyball (beach and indoor) in predominately repeated effort sports.

\section{RESULTS}

A total of 273 athletes gave informed consent, answered questionnaires and had ECGs performed. Female athletes represented $48.7 \%$ of the cohort with $92 \%$ athletes identified as Caucasian. The mean age was 18.9 years (median 18, IQR 14-36). The length of time spent in a high performance sport programme was a mean of 2.3 years (median 2, IQR $1-12$ ). The number of entrance athletes (length of time in high performance programme less than 1 year) was 128 (46.9\%). The classification of type of sports showed that $48.7 \%$ participated in repeated effort sports, $37.7 \%$ in continuous and $13.6 \%$ in static (table 1).

In response to the questionnaire, $71.1 \%$ returned no positive answers to the cardiac questionnaire $(n=194), 20.5 \%$ had one positive answer $(n=56)$ and $8.4 \%$ had two or more positive answers $(n=23)$. Asthma was self-reported in $17.9 \%$ of the athletes $(n=49)$.

The number of athletes having a normal ECG (Group 0) was $31 \%(n=83)$ and included isolated Sinus arrhythmia $(n=17)$. Group 1 changes were seen in 63\% $(n=173)$. These changes included bradycardia $(n=98)$, sinus arrhythmia $(n=61)$, atrioventricular block $(n=29)$, ECG criteria for left ventricular hypertrophy $(n=23)$, incomplete right bundle branch block $(n=46)$ and early repolarisation $(n=34)$. Group 2 changes were seen in $6.2 \%$ of athletes $(n=17)$. The most consistent finding in Group 2 was deep $\mathrm{T}$ wave inversion in the precordial leads, which was seen isolated in 10 athletes. The Group 2 athletes with sport, age, length of time in high performance programme and associated ECG findings are presented in table 2. Some of the more common ECG findings are shown in online supplementary figures 2-4. All Group 2 athletes had subsequent cardiac investigation. Echocardiograms in this group were normal with no structural abnormality found. For patients with rhythm abnormalities, Holter monitors did not show pathological changes.

Analysis of the type of exercise and duration of training demonstrated results given in table 3 . An increased length of time the athlete was in a high-performance programme, the higher likelihood the athlete had Group 1 ECG changes when compared with Group $0(p=0.039)$. This finding is perhaps expected as Group 1 changes are classical training-related findings as we considered the training more likely to induce heart alterations classified as Group 1 (training related ECG changes) than age. When considering multinominal logistic regression after adjusting for age and ranked on one-way analysis of covariance, this finding was not significant $(\mathrm{p}=0.19)$. 
Table 2 ECG classification of abnormal Group 2 changes

\begin{tabular}{|c|c|c|c|c|c|c|c|c|c|c|c|}
\hline Age & Years & Sex & Sport & $\begin{array}{l}\text { Junc } \\
\text { Tachy }\end{array}$ & TWI & $\begin{array}{l}R \\
\text { BBB }\end{array}$ & RVH & LAE & RAE & $\begin{array}{l}\mathbf{R} \\
\text { axis }\end{array}$ & $\begin{array}{l}\mathrm{L} \\
\text { axis }\end{array}$ \\
\hline 21 & 1 & $M$ & Trampoline & 1 & & & & & & & \\
\hline 17 & 2 & $\mathrm{~F}$ & Netball & & 1 & & & & & & \\
\hline 17 & 4 & $\mathrm{~F}$ & Basketball & & 1 & & & & & & \\
\hline 17 & 4 & $M$ & Basketball & & 1 & * & & & & & \\
\hline 17 & 2 & $\mathrm{~F}$ & Netball & & 1 & * & & & & & \\
\hline 16 & 1 & $M$ & Volleyball & & * & * & 1 & & 1 & & \\
\hline 19 & 1 & $\mathrm{~F}$ & Hockey & & 1 & * & & & & & \\
\hline 16 & 1 & $F$ & Netball & & 1 & * & & * & & & \\
\hline 33 & 5 & $M$ & Rowing & & & * & & 1 & & * & \\
\hline 25 & 7 & $\mathrm{~F}$ & Rowing & & 1 & & & & & & \\
\hline 23 & 5 & M & Cycling & & 1 & & & & & & \\
\hline 20 & 1 & $\mathrm{~F}$ & Rowing & & 1 & & & & & & \\
\hline 15 & 1 & M & Kayaking & & 1 & & & & & * & \\
\hline 22 & 2 & $\mathrm{~F}$ & Swim & & & 1 & & & & & 1 \\
\hline 20 & 2 & M & Cycling & & * & & 1 & & & & \\
\hline 20 & 4 & M & Kayaking & & * & * & 1 & & & & \\
\hline 20 & 4 & $M$ & Cycling & & & * & 1 & & & & \\
\hline
\end{tabular}

Years refer to number of years in high performance programme.

Trampoline classified as Static Sport, Netball/Basketball/Field Hockey/Volleyball as Intermittent, Cycling/Kayaking/Rowing as Continuous.

* Refers to the person having these findings in addition, but each athlete classified to interpreted principal finding.

An increased length of time the athlete was in a high-performance programme demonstrated that a higher likelihood the athlete had Group 2 ECG changes when compared with Group 0 and $1(p=0.046)$. However, when this was adjusted for age and ranked on one-way analysis of covariance the finding was not significant $(\mathrm{p}=0.16)$.

Continuous sports were less likely to have normal ECGs and more likely to have common and training-related ECG changes ( $n=96$ : Group 0, $n=20 ;$ Group 1, n=76; $\mathrm{p}<0.01)$. A repeated effort sport ( $n=124$ : Group 0, $n=44$; Group 1, $n=80$ ) demonstrated there is 50\% less likelihood to have common and training-related ECG changes when compared with a continuous effort sport $(\mathrm{RR}=0.48)$. These differences remained after adjusting for possible confounders such as age and length of time in high performance programme (age, $\mathrm{p}=0.006$, length of time on programme, $\mathrm{p}=0.003$ ).

Sports requiring high VO2max (continuous and repeated effort sports) was more likely to be in Group 1 (the common and training-related ECG change) than in Group 0 (RR 2.7, $\mathrm{p}=0.01)$. This finding was not influenced greatly by age (RR 2.9) or length of time in high performance programme (RR 2.7). Athletes in sports requiring high VO2max were 4.75 times more likely to have Group 2 (uncommon and training unrelated) ECG changes but this was not statistically significant $(p=0.14)$. Age (RR 5.0) and length of time in a high performance programme (RR 4.7) did not alter this relative risk to any great extent.

With respect to the symptom questionnaire, a single yes to any of the questions was not associated with any type of ECG changes (Group 0, 1 or 2). However, answering yes to two or more questions was associated with having a non-normal ECG (both Group 1 and Group 2) and this was statistically significant $(p=0.04)$. When considered by ECG groups, athletes with symptoms were seen in Group $0(n=16$ of 83$)$, Group $1(n=34$ of 173$)$ and Group $2(n=5$ of 17$)$ and this was not statistically significant. The questionnaire did not help in detecting athletes with unexpected and training unrelated ECG changes.
Table 3 ECG findings classified by 2010 ESC groups for all the respective analysed groups

\begin{tabular}{|c|c|c|c|}
\hline Category & Group 0 & Group 1 & Group 2 \\
\hline All athletes, $n=273$ & $83(31 \%)$ & $173(63 \%)$ & $17(6.2 \%)$ \\
\hline Entrance level (128) & 46 & 77 & $5(3.9 \%)$ \\
\hline$>1$ year in programme (145) & 37 & 96 & $12(8.3 \%)$ \\
\hline $\begin{array}{l}\text { High maximal uptake } \\
\text { (V02max) sport (236) }\end{array}$ & 64 & 156 & $16(6.8 \%)$ \\
\hline Static sport (37) & 19 & 17 & $1(2.7 \%)$ \\
\hline $\begin{array}{l}\text { High V02max - continuous } \\
\text { (103) }\end{array}$ & 20 & 76 & 9 \\
\hline $\begin{array}{l}\text { High V02max-repeated } \\
\text { effort (133) }\end{array}$ & 44 & 80 & 7 \\
\hline $\begin{array}{l}\text { Entrance and High V02max } \\
\text { sport (106) }\end{array}$ & 35 & 66 & 5 \\
\hline Entrance and Static sport (20) & 9 & 10 & 0 \\
\hline $\begin{array}{l}>1 \text { year in programme and } \\
\text { high V02max sport (130) }\end{array}$ & 29 & 90 & 11 \\
\hline $\begin{array}{l}>1 \text { year in programme and } \\
\text { Static sport }{ }^{17}\end{array}$ & 10 & 7 & 0 \\
\hline
\end{tabular}

$\begin{array}{lccc}\begin{array}{l}\text { Entrance and High V02max } \\ \text { sport-continuous (46) }\end{array} & 12 & 32 & 2 \\ \begin{array}{l}\text { Entrance and High V02max- } \\ \text { repeated effort (62) }\end{array} & 24 & 35 & 3 \\ \begin{array}{l}>1 \text { year in programme } \\ \text { and high V02max sport- } \\ \text { continuous (57) }\end{array} & 8 & 44 & 5(8.7 \%) \\ \begin{array}{l}>1 \text { year in programme and } \\ \text { high V02max-repeated } \\ \text { effort (71) }\end{array} & 20 & 45 & 6(8.4 \%)\end{array}$

Continuous=performance occurs at a high percentage of maximal oxygen uptake with predominately a continuous consistent effort, (2) Repeated effort=performance occurs at a high percentage of maximal oxygen uptake with repeated sprinting and/or jumping efforts and (3) Static=performance occurs at a lower percentage of maximal oxygen uptake and generally of a static nature.

\section{DISCUSSION}

The principal aim of this study of assessing and categorising the ECG findings in a group of young Australian high-performance athletes with respect to the ESC 2010 guidelines demonstrated an incidence of abnormal and training unrelated (Group 2) ECG changes of $6.2 \%$. This is in line with previous studies of predominately Caucasian populations. ${ }^{13} 14$ Athletes with a short training time in high performance programme $(<1$ year) had an abnormal ECG rate of $3.9 \%$ consistent with other studies of this nature from other populations in the same age group. ${ }^{9} 14$ As these Group 2 ECG findings are considered to be training unrelated, it would not be considered that more training, duration and intensity would result in a higher incidence of athletes in this ECG change group. Following from this, the group with the highest rate of abnormal ECGs (Group 2) occurred in $8.3 \%$ of athletes where performance occurs at a high percentage of maximal oxygen uptake and the athletes had been in a high performance programme for greater than 1 year $(n=12$ of 145 athletes greater than 1 year high performance training compared with $\mathrm{n}=5$ of 128 entrance level athletes).

We demonstrate an association between an increased length of time in a high-performance programme and the number of athletes that had Group 2 ECG changes. With specific sport 
classifications, athletes with performance at high VO2max demonstrated the unexpected finding of a $4.75 \times$ increase in likelihood of Group 2 ECG changes. This finding was statistically insignificant (posthoc analysis demonstrated that the study was underpowered by 44 athletes). It is not expected that Group 2 ECG changes would be related to length of time in programme nor would it be expected that sports with high VO2max being more highly represented compared with static sports. A possible hypothesis is that some of the uncommon and training unrelated ECG changes may be due to the normal remodelling of the athletes' heart and not due to underlying pathology. When athletes enter a high-performance programme at the South Australian Sports Institute, there is a large and progressive increase in their volume and intensity of training. Heart remodelling can be considered as highly likely due to this reason. For high VO2max sports combined with increased training, heart remodelling with training can also be considered highly likely.

Increasing age of the athlete and length of time in a high-performance programme are obviously linked. However, age can only formally be considered to be a confounder if it is associated with the length of time in the high-performance which it is not. Age is therefore a covariate. Additionally, when ECG changes, age and length of time in a high-performance programme are analysed with multinomial logistic progression, there is little alteration to the other coefficients in the model in comparison to the non-adjusted model, demonstrating that age and length of time are not confounders.

The principal Group 2 finding in our study was $\mathrm{T}$ wave inversion extending beyond precordial leads. It is well established that teenage athletes have a higher incidence of anteroseptal $\mathrm{T}$ wave inversion (a Group 2 classification in the ESC 2010 guidelines) in the precordial leads. ${ }^{13} 14$ Thus, it would be expected that as age increased the number of athletes with this particular ESC classification, Group 2 finding would diminish. In our study, the numbers with precordial $\mathrm{T}$ wave inversion increased as athlete age increased. In other words, as there is an increased length of time in the programme and with concomitant increasing age you would expect that the number of Group 1 (common and training-related) ECG changes would increase, which was demonstrated, but you would also expect that the number of Group 2 (uncommon and training unrelated) ECG changes would decrease due to the maturation of the adolescent heart.

This study confirms the well known and understood premise that training induces cardiac changes and as such there was a demonstrable association between being in a high performance sport programme for an increased length of time with having common and training related ECG changes. An Australian study $^{15}$ looking at an elite cohort of professional and international athletes $86 \%$ had Group 1 changes, this cohort had a Group 2 abnormality rate of $17.3 \%$. This is higher than that reported in other studies of University athletes entering Dutch ${ }^{16}$ and American ${ }^{11}$ athletic systems.

In our study, we further classified sports where performance occurs at a high percentage of maximal oxygen uptake by the nature of the sport and its physiological demands-continuous or repeated efforts. Athletes performing at a high percentage of their maximal oxygen uptake in a continuous manner had 50\% more common and training-related ECG changes compared with athletes that performed at a high percentage of their maximal oxygen uptake in multiple repeated efforts. Static sports had much fewer ECG changes compared with sports where athletes performed at a high percentage of maximal oxygen uptake.

Previous studies have demonstrated that use of personal symptom and family history questionnaires alone are inadequate in identifying athletes that may have ECG abnormalities that predispose to SCD. ${ }^{8}$ In our study at first glance, the questionnaire appeared like it may be useful with athletes that responded with more positive answers had more ECG changes. However, when actual cardiac symptoms were analysed separately, this was not the case. Thus, our study also confirms the proposition that a screening questionnaire is not a useful tool in detecting which athletes will have abnormal ECG changes. We are unable to make a comment about the utility of a questionnaire to facilitate the risk stratification of SCD in this population. The asthma rate of $17.9 \%$ is comparable to other studies of high-performance athletes. ${ }^{17}$

By implementing a prospective cross-sectional study using the ESC 2010 guidelines, it can be seen in our study that the number of athletes with Group 2 changes (follow-up rate) increases with (1) undertaking a sport where performance is at a high percentage of maximal oxygen uptake and (2) an increased length of time that the athlete is in a high-performance programme. Further indirect evidence for this proposition is the study on high performance elite and international level athletes with 17.3\% having Group 2 changes. ${ }^{15}$ It is likely that some of the Group 2 ECG changes in the 2010 ESC guidelines will be proven to be related to athletic endeavours (training related).

There are several limitations of this study. Our study represents a relatively small cohort of elite athletes and overall numbers have meant that some of our findings are suggestive but not adequately powered for a definitive result. In addition, the group of athletes were predominately Australian Caucasian athletes and thus the findings may not be applicable to other ethnic groups including the Australian Indigenous and Polynesian populations of the Australasian region. As the study is cross-sectional rather than longitudinal, it is not possible to know whether the Group 2 ECG changes attributed to training (taking into account type of sport and length of time in sport) is borne out over time. A study of athletes with normal ECGs at entry to training and repeated at the end of a training programme may further clarify this.

\section{CONCLUSION}

This study demonstrated that the number of athletes with Group 2 changes (using 2010 ESC guidelines) was dependent on the length of time in a high performance programme. High VO2max sport participation was associated with Group 1 ECG changes and had a high proportion of the type 2 ECG changes (15 out of the 16 Group 2 changes) but this did not attain statistical significance. Overall, the further investigation rate was $6.2 \%$ consistent with other studies.

Contributors All authors in this study have made substantial contribution to conception and design, acquisition of data or analysis and interpretation of data; drafting the article or revising it critically for important intellectual content and final approval of the version to be published.

Funding The authors have not declared a specific grant for this research from any funding agency in the public, commercial or not-for-profit sectors.

Competing interests None declared.

Patient consent for publication Not required.

Provenance and peer review Not commissioned; externally peer reviewed.

\section{REFERENCES}

1 Maron BJ, Bodison SA, Wesley YE, et al. Results of screening a large group of intercollegiate competitive athletes for cardiovascular disease. J Am Coll Cardiol 1987; 10:1214-21. 
2 Maron BJ, Thompson PD, Puffer JC, et al. Cardiovascular Preparticipation screening of competitive athletes. Circulation 1996;94:850-6.

3 Borjesson M, Dellborg M. Is there evidence for mandating electrocardiogram as part of the pre-participation examination? Clin J Sport Med 2011;21:13-17.

4 Drezner J. Corrado D: examination? Clin J Sport Med 2011;21:18-24.

5 Bjornstad H, Smith G, Steorstein L, et al. Electrocardiographic and echocardiographic findings in top athletes, athletic students and sedentary controls. Cardiology 1993:82:66-74.

6 Drezner JA, Fischbach P, Froelicher V, et al. Normal electrocardiographic findings: recognising physiological adaptations in athletes. Br J Sports Med 2013;47:125-36.

7 Pelliccia A, Culasso F, Di Paolo FM, et al. Prevalence of abnormal electrocardiograms in a large, unselected population undergoing pre-participation cardiovascular screening. Eur Heart J 2007;28:2006-10.

8 Wilson MG, Basavarajaiah S, Whyte GP, et al. Efficacy of personal symptom and family history questionnaires when screening for inherited cardiac pathologies: the role of electrocardiography. Br I Sports Med 2008;42:207-11.

9 Hevia AC, Fernández MM, Palacio JMA, et al. ECG as a part of the preparticipation screening programme: an old and still present international dilemma. Br I Sports Med 2011:45:776-9
10 Corrado D, Pelliccia A, Heidbuchel $H$, et al. Recommendations for interpretation of 12-lead electrocardiogram in the athlete. Eur Heart J 2010;31:243-59.

11 Weiner RB, Hutter AM, Wang F, et al. Performance of the 2010 European Society of cardiology criteria for ECG interpretation in athletes. Heart 2011;97:1573-7.

12 Mitchell JH, Haskell W. Snell P and van cAMP S: Task Force 8: classification of sports. JCAC 2005;45:1364-7.

13 Papadakis M, Basavarajaiah S, Rawlins J, et al. Prevalence and significance of T-wave inversions in predominantly Caucasian adolescent athletes. Eur Heart J 2009;30:1728-35.

14 Sharma S, Whyte G, Elliott P, et al. Electrocardiographic changes in 1000 highly trained junior elite athletes. Br J Sports Med 1999;33:319-24.

15 Brosnan M, LeGerche A, Kalman J, et al. The Seattle criteria increase the specificity of preparticipation ECG screening among elite athletes. BrJSportsMed 2013.

16 Bessem B, Groot FP. Nieuwland W: the Lausanne recommendations: a Dutch experience. BrJSportsMed 2009;43:708-15.

17 Turmel J, Poirier P, Bougault V, et al. Cardiorespiratory screening in elite endurance sports athletes: the Quebec study. Phys Sportsmed 2012;40:55-65. 